\title{
Corela
}

Cognition, représentation, langage

HS-35 | 2022

Anaphore et pronoms en anglais : convergences, différences et complémentarité de quelques approches linguistiques

\section{Les pronoms non anaphoriques : perspectives de grammaire générative transformationnelle}

\section{Anne Jugnet}

\section{OpenEdition}

Journals

Édition électronique

URL : https://journals.openedition.org/corela/14309

DOI : 10.4000/corela.14309

ISSN : 1638-573X

Éditeur

Cercle linguistique du Centre et de l'Ouest - CerLICO

Référence électronique

Anne Jugnet, « Les pronoms non anaphoriques : perspectives de grammaire générative

transformationnelle », Corela [En ligne], HS-35 | 2022, mis en ligne le 04 janvier 2022, consulté le 24 janvier 2022. URL : http://journals.openedition.org/corela/14309 ; DOI : https://doi.org/10.4000/ corela.14309

Ce document a été généré automatiquement le 24 janvier 2022.

\section{c) (i) (2)}

Corela - cognition, représentation, langage est mis à disposition selon les termes de la licence Creative Commons Attribution - Pas d'Utilisation Commerciale - Partage dans les Mêmes Conditions 4.0 International. 


\title{
Les pronoms non anaphoriques : perspectives de grammaire générative transformationnelle
}

\author{
Anne Jugnet
}

\section{Introduction}

1 Les approches génératives transformationnelles classiques présentent rarement un traitement commun à l'ensemble des pronoms. Une distinction peut être faite entre les pronoms anaphoriques et les autres (i.e. les pronoms wh- ou les explétifs). La distribution des pronoms anaphoriques a fait l'objet de nombreux travaux en syntaxe générative; cette question était l'un des sujets centraux d'un cadre théorique, i.e. la théorie du gouvernement et du liage, cadre développé dans les années 1980 (dans la lignée de Chomsky 1981 et ses Lectures on Government and Binding). Dans ces travaux étaient discutés des principes permettant de prédire ou expliquer la distribution des pronoms réfléchis et réciproques (himself, each other) par opposition aux pronoms anaphoriques (tels que him) ou des expressions référentielles (telles que Chomsky's followers). Les pronoms non anaphoriques n'ont quant à eux pas fait l'objet d'analyses similaires, ce qui peut s'expliquer par le caractère hétérogène de cet ensemble : parmi les pronoms non anaphoriques figurent différents pronoms wh-, mais aussi des explétifs (ou pronoms impersonnels). Ces deux sous-catégories soulèvent des problèmes distincts, et seront examinées successivement.

\section{Les grammaires transformationnelles classiques: un traitement uniforme des pronoms wh-?}

2 Tous les pronoms wh-sont supposés partager des propriétés syntaxiques : ils ne sont généralement pas in situ (dans la position attendue, habituellement associée à leur fonction, par exemple la position qui suit le verbe pour un objet) mais sont réalisés en 
tête d'énoncé (périphérie gauche). Dans les approches générativistes transformationnelles classiques, les phrases comprenant un pronom wh- ne sont donc pas des structures canoniques, la structure de surface diffère de la structure profonde. Ces phrases impliquent ainsi une transformation, plus précisément un mouvement wh- : de la position en structure profonde, correspondant à la fonction associée, le pronom wh- est déplacé vers la périphérie gauche. L'enjeu est alors de déterminer la position d'arrivée des pronoms wh- : s'agit-il d'une même position, que le pronom soit interrogatif, relatif, ou relatif libre? Certains auteurs (dont Haegeman 1991) soulignent les parallèles entre questions wh-, relatives et relatives libres : ces structures auraient la même syntaxe interne. On observerait donc un même mouvement dans les interrogatives $(2,9)^{1}$, pseudo-clivées (1), ou relatives (8):

(2) What is it $[t]^{2}$ that drives you in your life today ...?

(9) The real question, which is, what [t]'s the difference in somebody's life if you

look at somebody like those people that you've given everything to?

(1) what I do [t] ... is coach people

(8) whether it be your business, your contribution to society, money - whatever it is

[t] for you - your body, your family.

3 L'hypothèse est que tout mouvement est associé à une trace, et le mouvement whcorrespond à un mouvement $\mathrm{A}^{\prime}$ (angl. A-bar, fr. A-barre), c'est-à-dire un mouvement vers une position qui n'est pas associée à une fonction grammaticale déterminée (contrairement à ce qui est observé avec la passivation par exemple: au passif la position d'arrivée - de l'objet de la proposition à l'actif - est associée à la fonction sujet).

4 Une question qui a fait l'objet de nombreuses études, mais sur laquelle nous ne nous attarderons pas, est celle des limites et contraintes sur le mouvement: jusqu'où un élément wh- peut-il être séparé de sa position d'origine? Etant donné que des mouvements à longue distance sont observés (e.g. Who does Bob think that Mary believes that Mo loves), il paraît à première vue envisageable que les mouvements soient non bornés (unbounded). Toutefois Ross (1967) remarque que le mouvement est soumis à des contraintes : l'impossibilité d'un ensemble de structures peut être expliquée en termes de contraintes d'îlots (certains constituants, tels que des subordonnées wh-, des relatives, ou des subordonnées compléments de noms, ne permettant pas d'extractions). Une hypothèse souvent défendue est alors celle d'un mouvement cyclique, par étapes. Chomsky (1973) postule ainsi une chaîne d'étapes locales successives dans la dérivation. Le mouvement wh- est alors conçu comme une série de mouvements cycliques successifs de COMP à COMP ; ces mouvements étant donc soumis à la condition de localité (Subjacency Condition). Nous ne détaillerons pas ici l'ensemble des contraintes observées, car l'objectif de cette présentation est l'analyse d'exemples attestés dans un texte : nous ne nous attarderons ainsi pas sur les contraintes décrites en grammaire transformationnelle standard, mais examinerons plutôt sur les questions soulevées par les exemples du texte.

5 L'hypothèse d'un mouvement wh- uniforme vers une position A' (non argumentale), quelle que soit la fonction du constituant et quel que soit le type de structure (interrogative, relative), peut être affinée. Les exemples du texte appelant un traitement différencié sont ainsi examinés dans les sections suivantes. 


\subsection{Différentes structures interrogatives?}

6 Les structures interrogatives directes pourraient ne pas recevoir d'analyse unique, mais pourraient plutôt correspondre à deux structures, selon que le constituant wh- est sujet ou non. En effet, lorsque le pronom wh- est sujet, le mouvement opère à vide : il n'est pas observable si l'on s'en tient à la simple suite linéaire. Quelle analyse peut alors recevoir l'exemple (2) ?

(2) What is it that $[t]$ drives you in your life today ...?

7 Doit-on postuler que le pronom wh- est en position de sujet (sœur du VP), ou plutôt en position de spécifieur du CP, comme les autres formes en wh-?

8 La question n'est toujours pas tranchée. Certains, comme Trotta (2004), dans la lignée de Chomsky (1986), postulent que le pronom wh- sujet est dans sa position de sujet (Spec,IP). Cette analyse est justifiée par le fait que la structure ne comprend pas d'indice de mouvement: les questions portant sur le sujet n'observent pas l'inversion sujet-auxiliaire (n'impliquent donc pas d'insertion éventuelle de do). D'autres, comme Cheng (1991) soutiennent qu'il faut postuler un mouvement wh-car c'est ce mouvement qui permet la classification et ainsi l'identification de ce type de propositions. Cheng (1991) rejoint ainsi Huddleston (1984), pour qui un avantage de l'hypothèse d'un mouvement vide est qu'elle permet une analyse uniforme (et plus élégante) des propositions wh-. Ces considérations générales mises à part, certains arguments peuvent être avancés en faveur d'une analyse en termes de mouvement. Selon Trotta, le fait que that peut apparaitre après wh-pour introduire la proposition est un indice d'un mouvement de wh-sujet :

1. It will probably be evident from the field which of the players that are feeling the

heat most. (Radford 1988: 500)

2. I wonder if he could describe to us what influence that has been brought to bear

on the the [sic] overall calculation. $(\mathrm{BNC})^{3}$

9 Ainsi un traitement uniforme serait motivé et l'absence de l'auxiliaire do serait expliquée par l'hypothèse que do n'est pas nécessaire pour que le verbe soit en deuxième position - l'insertion de do étant conçue comme un dernier recours. Une analyse uniforme des différentes structures interrogatives est ainsi envisageable.

10 Comme nous l'avons déjà indiqué, le texte comprend des interrogatives comme des relatives (9), y compris des relatives libres $(1,8)$ : une analyse similaire de cet ensemble d'exemples est-elle motivée? Nous nous concentrerons sur les similitudes et différences entre interrogatives et relatives libres, car ce point a suscité plus de débats.

\subsection{Relatives libres vs. interrogatives}

11 L'analyse des relatives libres a fait l'objet de controverses: certains auteurs ont souligné un parallèle entre relatives libres et interrogatives, et en ont déduit que le relatif libre est en position de (Spec,CP), tandis que d'autres ont souligné leurs différences, et postulé que le relatif est en tête de relative.

12 En s'appuyant sur la syntaxe externe des relatives libres, certains auteurs (dont Bresnan \& Grimshaw (1978)) ont défendu l'idée que les relatives libres se démarquent des interrogatives, et postulé que le relatif est en position de tête de relative (« hypothèse de tête " (Head Hypothesis)). Cette hypothèse s'appuie sur des différences de syntaxe externe : le pronom wh- est relié à une trace dans la relative libre mais doit 
aussi être sélectionné par le prédicat de la proposition enchâssante (d'où l'idée que le pronom wh- est à la fois la tête du constituant et l'antécédent d'une trace). L'hypothèse de tête est motivée par des effets de correspondances (matching effects) : la catégorie de l'ensemble de la relative est déterminée par l'élément qui l'introduit, et doit correspondre à la catégorie de compléments habituellement sélectionnés par le prédicat enchâssant, une relative libre introduite par un pronom wh- est donc de catégorie nominale (et non propositionnelle) :

3. We should interview the woman with whom he goes out.

4. *We should interview with whoever he goes out. ${ }^{4}$

5. We should talk to the woman whom he dates.

6. We should talk to whom/whoever he dates.

7. We should talk to the woman with whom he goes out.

8. *We should talk to with whom he goes out.

Comme le montrent les exemples ci-dessus, empruntés à Riemsdijk (2006), dans le cas des relatives libres, les contraintes catégorielles imposées par le verbe enchâssant et le verbe enchâssé doivent correspondre. Ce qu'explique facilement l'« hypothèse de tête » : le constituant wh- est en position de tête et doit donc satisfaire les contraintes de sélection, comme les têtes habituelles. L'une des limites de cette approche est qu'elle rend moins facilement compte de la syntaxe interne des relatives libres - ce qu'ont souligné les partisans de l'analyse des relatives libres comme étant similaires à des propositions (relatives) habituelles.

Une autre hypothèse a donc été défendue, selon laquelle le pronom wh- est en position de spécifieur du complémenteur ( $\mathrm{Spec}, \mathrm{CP})$. Cette hypothèse, défendue en particulier par Groos \& Riemsdijk (1981), s'appuie essentiellement sur l'extraposition des relatives en allemand et en néerlandais, et sur la syntaxe interne des relatives libres (en anglais), plus précisément l'absence de complémenteur dans les relatives libres (lorsque la position relativisée n'est pas sujet). En effet, en allemand, si un mouvement en fin d'énoncé est opéré, c'est le relatif libre avec l'ensemble de la relative qui est déplacé, et non la proposition qui suit le relatif libre.

9. *Der Hans hat was zurückgegeben, er gestohlen hat.

10. Der Hans hat $[t]$ zurückgegeben, was er gestohlen hat.

Ainsi les relatives libres ne fonctionnent pas comme des relatives avec antécédent: dans ce dernier cas la relative seule est extraposée, l'antécédent restant dans sa position initiale :

11. Der Hans hat das Geld, das er gestohlen hat, zurückgegeben.

12. Der Hans hat das Geld zurückgegeben, das er gestohlen hat. ${ }^{5}$

Selon Bresnan \& Grimshaw (1978), ces différences peuvent être expliquées par le fait que les relatives libres impliqueraient une proposition réduite (IP et non CP). Mais il s'agit d'une hypothèse ad hoc sans laquelle il serait difficile de rendre compte de ces données dans leur approche.

Par ailleurs, selon Riemsdijk (2006), l'hypothèse d'un relatif en position de complémenteur permet de mieux rendre compte de la distribution des relativiseurs. En effet, si wh- est en position de tête comme le supposent Bresnan \& Grimshaw (1978), ce qui suit doit être une relative, donc un $\mathrm{CP}$, et les mêmes contraintes devraient être observées dans les relatives à antécédent et dans les relatives libres. Or ce n'est pas le cas, comme le montrent les contrastes d'acceptabilité dans les exemples suivants (empruntés à Riemsdijk 2006) :

13. I put the pie which/*which that/ that/ Ø you prepared in the refrigerator. 
14. I put [ _ ] what/ ${ }^{*}$ what that $/{ }^{*}$ that $/{ }^{*} \varnothing$ you prepared in the refrigerator. ${ }^{6}$

15. The pie which/ ${ }^{*}$ which that/ that $/{ }^{*} \varnothing$ was in the refrigerator tasted bad.

16. [ _ ] What $/{ }^{*}$ what that $/ *^{*}$ that $/{ }^{*} \varnothing$ was in the refrigerator tasted bad.

facilement de rendre compte de la syntaxe externe des relatives (et des effets de correspondance expliqués par l'hypothèse de tête défendue par Bresnan et Grimshaw (1978)).

19 Ainsi la syntaxe externe des relatives libres introduites par un pronom wh- les rapproche de syntagmes nominaux, alors que leur syntaxe interne les rapproche de propositions wh- habituelles.

Un débat similaire concerne les relatives en -ever: doivent-elles être analysées comme des relatives libres habituelles, ou doit-on plutôt postuler une structure spécifique, à part?

\subsection{Relatives en -ever vs relatives libres ?}

La plupart des auteurs postulent que les relatives en -ever sont similaires aux relatives libres habituelles (Alexiadou et al. 2000). Un parallèle pourrait donc être établi entre (1) et (8) :

(1) what I do ... is coach people

(8) whether it be your business, your contribution to society, money - whatever it is

for you - your body, your family

Toutefois ces deux formes ne sont pas interchangeables (notamment dans notre texte: whatever ne pourrait remplacer what en (1), et inversement what ne pourrait être substitué à whatever en (8)), et leurs distributions ne sont pas identiques. Ces différences pourraient motiver la remise en question par Donati et Cecchetto (2011) du parallèle habituellement admis entre relatives libres et relatives en -ever. Ces auteurs affirment en effet que les relatives introduites par un relatif en -ever ne sont pas des relatives libres habituelles, mais se rapprochent de relatives avec une tête (headed relative clauses). En d'autres termes, les relatives en -ever seraient similaires à des relatives avec antécédent. L'élément en -ever ne subirait pas de mouvement mais serait généré en tête de constituant (comme l'antécédent d'une relative) et les relatives en ever seraient ainsi des « pseudo-relatives libres ».

L'hypothèse de Donati et Cecchetto (2011) repose sur cinq arguments principaux. Caponigro (2018) a critiqué la validité de chacun. Ces arguments et leurs critiques sont rappelés ici.

Pour Donati \& Cecchetto (2011), les éléments en -ever se distinguent des relatives libres en ce qu'ils peuvent avoir un « emploi absolu» :

17. I can eat whatever is in the fridge.

17 '. I can eat whatever.

18. I can eat what is in the fridge.

$18^{\prime}$. *I can eat what.

Selon Donati et Cecchetto (2011), ces données montrent que les éléments en -ever sont des DPs quantificationnels, syntaxiquement distincts des autres expressions wh- (ils ne subiraient pas de mouvement). Caponigro (2018) oppose à ces données un argument diachronique : les emplois absolus sont apparus plus tardivement que les emplois dans les relatives libres, ce qui serait inattendu si les éléments en -ever étaient nominaux 
quantificationnels (pouvant optionnellement être des têtes de relatifs). Ces éléments seraient donc plutôt de véritables expressions wh- ; il est donc attendu qu'ils introduisent des propositions, leur emploi absolu étant un développement ultérieur dans lequel l'élément en -ever peut s'employer sans son argument propositionnel. Par ailleurs, selon Donati et Cecchetto (id.), contrairement aux relatives libres habituelles, les relatives en -ever permettent des relativiseurs (complémenteur that (argument 2) et pronoms relatifs (argument 3)) : elles se rapprochent ainsi de relatives avec antécédents. Le contraste suivant justifie selon eux la distribution de that :

19. You can read whatever books (that) are on the table.

20. *You can read what books that are on the table.

Or selon Caponigro (2018), dans la majorité des cas, la distribution de that est très différente dans les relatives en -ever et dans les relatives à antécédent: ainsi, les relatives à antécédent requièrent that si le relatif est sujet (ce qu'indique l'agrammaticalité de l'exemple 22), alors que that n'est pas requis dans le constituant wh- sujet des relatives libres, en -ever ou non: la présence de that n'est possible que si un nom intervient après le mot wh- (ce qu'illustrent 19 et 24).

21. You can read any book that's on the table.

22. *You can read any book 熙 is on the table.

23. You can read what's/*that's on the table.

24. You can read whatever (*that) is on the table.

De plus, that est exclu avec un autre relatif en -ever :

25. I can talk to whoever (*that)'s on the phone.

Caponigro (id.) en conclut que les formes en -ever sont plutôt similaires à des relatives libres.

31 Concernant la possible présence de relatifs après le constituant en -ever :

26. Whatever books for which she writes reviews are likely to become bestsellers.

27. *What books for which she writes reviews are likely to become bestsellers).

Selon Caponigro, les exemples (26-27) cités par Donati et Cecchetto (2011) relèvent d'un schéma très limité, non productif. Comme l'illustre (26), la présence d'un relatif dans la proposition suivant un relatif en -ever n'est possible que dans certains des cas où whatever est un déterminant - mais pas dans tous (28) ; par ailleurs, lorsque le pronom en -ever n'est pas déterminant, la présence d'un autre relatif est impossible (ce qu'illustrent 29 et 30, également empruntés à Caponigro (2018)) :

28. I'll talk to whatever students (*who) are problematic.

29. I'll talk to whoever (*who) is problematic.

30. You can do it however (*in which) you want.

33 Une autre propriété des subordonnées en -ever est qu'elles peuvent avoir la même distribution que des adjoints :

31. Whatever you say, I won't change my mind.

32. *What you say, I won't change my mind.

Mais, selon Caponigro (2018), ces adjoints en wh-ever diffèrent des relatives libres en ever et devraient être analysées comme une catégorie de subordonnées à part. En effet, elles n'ont pas la même syntaxe, puisqu'elles apparaissent en position disloquée, elles ne sont pas arguments, et sont sémantiquement différentes: elles reçoivent une interprétation concessive (whatever peut être paraphrasé par no matter what). 

libres le peuvent selon Donati \& Cechetto (mais cette généralisation ne s'applique qu'à l'italien). Selon Caponigro, ces données sont discutables, et il n'existe pas de relatives libres infinitives.

d'adjoint des relatives en -ever en position argumentale, la plupart des relatives en -ever sont similaires à des relatives libres introduites par un pronom simple. En particulier, la relative dans le texte à analyser correspond à une relative libre habituelle (elle n'est pas un adjoint). Toutefois, contrairement aux relatives libres en -ever habituelles, elle est suivie par la liste d'individus de l'ensemble auquel la relative est associée. Selon Baker (1995), habituellement, une relative en -ever ne permet pas cette explicitation, comme le montrent ces exemples adaptés de Caponigro (2018):

33. He read what (books) she read, namely, Lolita and A Clockwork Orange.

$34 .{ }^{*}$ He read whatever (books) she read, namely, Lolita and A Clockwork Orange.

L'explicitation observée en (8) est peut-être due au fait que la relative apparaît dans un énoncé averbal, reprenant une relative libre dans l'énoncé précédent. Plus précisément, la relative libre dans le corpus (8) est indéfinie (selon la terminologie de Culicover \& Jackendoff (2005: 323)) ou non restrictive (selon la terminologie de Riemsdijk 2006) : ainsi, elle est comparable à un pronom (non référentiel) en any- tel que anything (cf. 4. we can rationalize anything), contrairement à une relative libre définie (paraphrasable par 'le x tel que p').

\section{Des approches génératives alternatives des pronoms wh-}

Si la grammaire générative traditionnelle explique la distribution des pronoms wh-en termes de mouvement, d'autres cadres théoriques génératifs ne font pas appel à l'idée d'une discordance entre structure profonde et de surface. La syntaxe doit alors se concentrer sur la description de l'agencement linéaire sans supposer de transformations, ni de vides ou traces dans la structure. Les structures impliquant un pronom wh-sont ainsi analysées en termes de dépendances (filler-gap dependencies) : la structure syntaxique est bien formée si le constituant dans lequel le pronom whapparaît est de même catégorie que la catégorie de l'argument sélectionné par le verbe. Ainsi, dans un exemple tel que (1), l'objet (nominal) du verbe n'est pas réalisé localement, en position d'objet, l'information (indication d'un vide nominal) est héritée par le syntagme verbal, et la phrase devient grammaticale seulement parce que ce constituant se combine finalement avec un constituant nominal : what.

(1) What I do _

Par contre, si un constituant avec un vide nominal se combine avec un constituant prépositionnel, aucune dépendance ne peut être établie, la discordance mène à l'agrammaticalité de l'ensemble.

$\left(1^{\prime}\right){ }^{*} \mathrm{To} /{ }^{*}$ On what I do depends on how things play out for the country.

Par ailleurs, dans une autre approche non transformationnelle, Culicover \& Jackendoff (2005 : 302) ne postulent pas non plus de mouvement A', et défendent l'idée d'une distinction entre la licence directe (avec une connexion locale) et la licence indirecte, où un constituant est réalisé dans une position non canonique (y compris dans une 
autre proposition, ex. Terry is flirting. - Yeah, with Sandy). Dans leur approche, dont le but est de limiter les éléments implicites dans les structures syntaxiques et les opérations implicites, Culicover \& Jackendoff (2005) réanalysent les structures résultant d'un supposé mouvement A-barre (dans les approches transformationnelles) comme des cas spéciaux de licence indirecte: le constituant "déplacé en tête d'énoncé » est considéré comme étant l'orphelin d'une licence indirecte, possible par un lien entre l'orphelin et la position cible dans l'antécédent. Un lien de type chaîne est postulé :

35. [With whom $]_{i}^{\text {ORPH }}$ is Terry flirting $t_{i}^{\text {TARG }}$. (Culicover \& Jackendoff 2005: 303)

41 La trace $" t_{i}$ " représente ici le vide syntaxique marquant la fonction de l'élément déplacé (et non la trace laissée par un mouvement), elle représente la position supposée du constituant s'il avait eu une réalisation canonique (licence directe).

Les approches génératives ne sont ainsi pas nécessairement transformationnelles, et les différents traitements des fonctionnements des pronoms wh- qui ont été proposés impliquent des partis pris théoriques marqués (l'hypothèse du mouvement étant l'une des plus contestées dans d'autres approches).

Un autre ensemble de pronoms non anaphoriques a fait l'objet d'analyses sujettes à débat (et remises en question dans d'autres approches) : les explétifs.

\section{Les autres pronoms non anaphoriques : les explétifs}

L'existence même des explétifs pose un problème théorique: selon un principe d'économie, l'emploi de tels pronoms devrait être évité, car les explétifs, dénués de contenu thématique, ne répondent pas aux exigences sélectionnelles de(s) prédicats. La question des raisons de leur emploi est donc souvent soulevée. L'idée que leur présence pourrait être motivée par des contraintes structurelles est souvent défendue. Les emplois de there et it sont ainsi rattachés à des contraintes syntaxiques, telles que la nécessité d'avoir une position sujet instanciée. Si le seul argument du verbe est réalisé en position postverbale, alors un explétif est requis : c'est le cas des verbes inaccusatifs, pour lesquels il est supposé que leur argument unique est généré en position objet, there remplissant alors la position sujet. C'est également le cas lorsque le sujet ("réel », ou sujet logique) est phrastique: de tels sujets sont généralement extraposés en position finale en raison de leur longueur et de leur complexité, l'explétif it remplissant alors la position de sujet syntaxique.

Ces contraintes expliqueraient donc la présence de there et it dans les exemples (10), (2) et (5) du texte à étudier :

(10) there was emotion that was there

(2) What is it that drives you in your life?

(5) when emotion comes into it, the wiring changes in the way it functions ${ }^{7}$

Toutefois, comme le notent Culicover \& Jackendoff (2005) la contrainte de position sujet obligatoirement instanciée n'est pas observée dans toutes les structures : ainsi, les infinitives contrôlées ne requièrent pas de sujet (To err is human). Pour Culicover \& Jackendoff (2005 : 196) la contrainte doit plutôt être reliée au fait que les fonctions grammaticales doivent être instanciées par un syntagme nominal dans une proposition (ce que Culicover et Jackendoff appellent le GF tier). Ainsi, dans le cas prototypique, la fonction grammaticale est doublement liée (à un argument sémantique et à une 
position syntaxique sujet), mais elle peut aussi être seulement liée sémantiquement (dans le cas des sujets contrôlés, ou lorsque la proposition n'a pas de sujet), ou seulement liée par la syntaxe (l'explétif n'exprimant pas un argument sémantique, dans des exemples tels que it's raining).

Une discussion plus détaillée des contraintes auxquelles les explétifs répondent est donc nécessaire. Etant donné que les deux explétifs sont traditionnellement analysés comme relevant de phénomènes distincts, ils seront examinés dans deux sections successives.

\subsection{There}

La plupart des travaux consacrés à there tentent non pas d'expliquer la présence de ce pronom (de justifier l'emploi d'un explétif plutôt qu'un sujet référentiel), mais plutôt d'expliquer les contraintes qui pèsent sur son emploi.

Le pronom there des constructions impersonnelles a traditionnellement été analysé comme formant une chaîne avec le nominal postverbal (Chomsky 1995, Perlmutter 1983, Burzio 1986, Postal \& Pullum 1988, Svenonius 2002) : there partage ainsi un indice avec l'élément postverbal. La question centrale est plutôt celle de sa sélection : l'enjeu est de délimiter syntaxiquement la classe de prédicats permettant un tel sujet impersonnel.

Plusieurs hypothèses ont été avancées, la plupart n'étant pas seulement syntaxiques (sans appel à des notions sémantiques). Quel type de contraintes sont en en jeu : s'agitil de restrictions de sélection imposées par des prédicats spécifiques (comme semble le supposer Levin 1993)? Ou ces contraintes sont-elles plutôt structurelles, reflétant la présence d'une tête fonctionnelle ? (Cette hypothèse est favorisée dans des versions plus récentes des théories génératives, comme l'approche minimaliste, dans lesquelles les représentations syntaxiques impliquent des projections fonctionnelles.)

51 Comme le rappelle Deal (2009), there est associé aux vPs dépourvus d'arguments externes et l'insertion de there est donc impossible avec verbes transitifs, inergatifs ${ }^{8}$, et inchoatifs9. Burzio (1986) avait ainsi postulé que l'insertion de there était possible seulement avec les inaccusatifs. Cette généralisation doit être nuancée: les verbes inaccusatifs ne peuvent pas tous avoir pour sujet there. Si assemble, circulate ou roll permettent there, d'autres inaccusatifs, tels que start, ne le permettent pas.

Selon Levin (1993), la distribution de there devait donc impliquer la considération de paramètres sémantiques : les inaccusatifs décrivant un changement d'état (tels que melt ou snow) se distinguent ainsi de simples inaccusatifs (tels que appear ou arrive) en ce que seuls ces derniers permettent there.

Deal (2009) rejette l'idée que la sémantique lexicale ou la classe lexicale influe sur la distribution de there. En effet, l'idée que there serait compatible avec des verbes inaccusatifs qui ne seraient pas des verbes de changement d'état (défendue par Levin 1993) n'est pas entièrement satisfaisante, car des contre-exemples sont facilement trouvés : disappear, exclu des verbes de changement d'état, ne permet pas there, alors que bloom, qui implique un changement d'état, permet there. L'hypothèse de Deal (2009 : 291) est donc que «le vP d'une racine verbale inaccusative peut contenir l'explétif there seulement s'il ne contient pas CAUSE » (nous traduisons). 
Ainsi selon Deal (2009), qui intègre des éléments d'analyses de Haegeman (1991) ou Hale \& Keyser (2000), la présence de there peut être expliquée par l'absence d'une tête fonctionnelle CAUSE, une structure fonctionnelle associée à certaines racines verbales. L'hypothèse d'un lien entre non-causativité et présence de there est déjà défendue par Haegeman (1991), puis Hale \& Keyser (2000). Haegeman (1991) avait ainsi remarqué que la plupart des verbes qui participent à l'alternance causative (tels que break, dry, open) ne permettent pas l'insertion de there:

36. Pat broke the window.

36 . The window broke.

$36 "$. *There broke the window.

La projection fonctionnelle serait possible seulement avec des vPs qui n'ont pas d'argument externe (ou d'argument événementiel). La tête CAUSE devrait être interprétée comme impliquant une cause de type événement (et non entité), et impliquant une relation causale directe.

Si elle est associée à une interprétation sémantique, cette tête doit être représentée en syntaxe (car elle est relativement indépendante de propriétés lexicales spécifiques). Cette présence en syntaxe est, selon Deal (2009), reflétée par sa compatibilité avec des modifieurs prépositionnels. Si la plupart des verbes peuvent être modifiés par des syntagmes prépositionnels introduits par from pouvant décrire une origine, seuls les prédicats associés à une tête CAUSE peuvent être modifiés par des syntagmes en from décrivant une cause.

37. The window cracked from the pressure.

38. The plane arrived \{from Boston / *from the tailwind\}.

De plus, le modifieur by itself reçoit une interprétation différente selon le contexte : en l'absence d'une tête CAUSE, il est généralement compris comme signifiant alone (glose possible en 39), alors que s'il est associé à un verbe incluant une tête CAUSE, il signifie 'sans cause externe', comme dans l'exemple 40 :

39. She arrived by herself. ( $\approx$ alone / $\neq$ without outside help or cause)

40. The window cracked by itself. ( $\neq$ alone / $\approx$ without outside help or cause)

Par ailleurs, la présence de la tête there est associée à une lecture stative du verbe : ex. There grew some corn in our garden last year (statif, présence de there possible) ; *There grew some corn very slowly in Massachussetts (dynamique, présence de there impossible), cf. Deal (2009: 296).

Selon Deal (2009), there apparaît bas dans la structure fonctionnelle, cette position basse est motivée par une relation locale entre there et le DP associé, relation qui joue un rôle central dans l'interaction de there avec les verbes à montée: l'accord local exclut des there surnuméraires, et permet ainsi de prédire l'agrammaticalité de phrases telles que *There seemed there to be a problem. Dans une approche locale, l'agrammaticalité de ce type d'énoncé est expliquée par la trop grande distance entre le there en position de sujet de matrice, trop éloigné de son associé (nominal). There serait donc, selon Deal (2009), généré dans la subordonnée, et non dans la position de spécifieur du verbe à montée. ${ }^{10}$

Quant à la motivation de l'emploi de there, elle n'est que peu décrite syntaxiquement (et n'est pas présentée comme relevant d'une contrainte syntaxique à proprement parler). Une hypothèse souvent soutenue est que l'emploi de l'explétif est justifiée par une contrainte de définitude et des considérations de structure informationnelle. En effet, la relation d'accord entre l'explétif et le DP implique que des traits soient portés par 
there : l'explétif n'est donc pas totalement dénué de traits sémantiques. En particulier, les syntagmes nominaux associés à there sont non spécifiques, et doivent être interprétés dans le syntagme verbal (qui correspond au domaine de clôture existentielle, selon Diesing 1992). La restriction de définitude observée en position sujet pourrait expliquer l'insertion de there : cette insertion permet à un argument indéfini de ne pas être réalisé en début de phrase, comme les sujets le sont habituellement en anglais. En d'autres termes, elle permet à l'argument indéfini de rester en position objet, dans la portée de la clôture existentielle. Elle permet alors à des sujets indéfinis de rester en bas de la structure, d'où une interprétation faible non ambiguë. La présence de there permettrait ainsi la présence d'un élément nouveau en discours (et potentiellement syntaxiquement " lourd ») en fin de proposition, ce qui obéit à des principes de structure informationnelle. L'insertion de there n'est donc pas entièrement expliquée en syntaxe, et implique la prise en compte de paramètres informationnels ou discursifs.

Comme on l'a vu, les types de contraintes sur la distribution de there ont pu faire l'objet de discussions. Toutefois ces débats n'impliquaient pas des enjeux théoriques aussi importants que les discussions sur la distribution et la motivation de l'explétif $i t$. Ces débats sont examinés dans la prochaine - et dernière - partie de cet article.

\subsection{It} générative transformationnelle classique. L'existence de l'explétif répond (pour la majorité des auteurs) à des contraintes non controversées (telles que le principe EPP ${ }^{11}$, selon lequel la position sujet doit être instanciée). Mais la présence d'explétifs en position de complément a été l'objet de controverses : certains auteurs ont affirmé que les explétifs peuvent apparaître dans des positions sous-catégorisées (telles que la position d'objet), s'opposant ainsi à l'hypothèse (généralement admise dans les approches chomskyennes) que la sélection syntaxique est nécessairement associée à une assignation de rôle sémantique. Postal et Pullum (1988) remettent ainsi en question la généralisation de Burzio (1986), selon laquelle seuls les verbes qui assignent un rôle thématique peuvent également assigner le cas à leur objet. L'existence d'idiomes tels que it rained mackerel remet en effet en question cette généralisation: dans ce ce type d'exemple, le cas est assigné à un objet syntaxique qui n'est pas sélectionné sémantiquement. Comme le notent Börjars \& Vincent (2008), diverses structures 
impliquent un décalage entre sélection syntaxique et sélection sémantique de l'objet (en d'autres termes, des syntagmes nominaux se voient assigner un cas mais ne reçoivent pas de rôle thématique dans un ensemble de structures): structures impliquant un objet interne (cognate object), telles que he laughed a cruel laugh, ou des pseudo-objets dans des structures résultatives (telles que he laughed his head off). De telles structures n'impliquent pas de sélection sémantique claire d'un objet syntaxique.

Dans leur critique de Burzio (1986), Postal et Pullum (1988) soutiennent que des explétifs peuvent être réalisés en position objet, ou complément, dans des structures extraposées (telles que he insisted upon it that he was being shadowed), ou encore dans des exemples similaires à notre exemple (5) (when emotion comes into it), exemples tels que beat it ou live it up. Selon eux, it est non référentiel dans ces idiomes, aucun syntagme ne pouvant le remplacer sans modifier le sens (comme le montrent l'inacceptabilité des gloses ??beat things ou ??live your life up). Cette analyse est remise en question par un ensemble de linguistes (qui défendent l'association nécessaire entre sélection syntaxique et assignation de rôle sémantique). Pour Rothstein (1995), le pronom it, dans des idiomes, a un référent vague, non spécifié, mais qui peut être explicité : par exemple, buy it pourrait être paraphrasé par buy the story. Selon Rothstein (1995: 527), moins l'emploi est idiomatique, plus il est facile de remplacer it par un objet non pronominal. Le remplacement est ainsi envisageable dans un ensemble de cas (have it out with = the matter, fight it out with = the matter, keep it up = the work/the appearance, rush it = the matter/things, buy it = buy the story, walk it = walk the distance, get it together = get your act together). Certaines gloses semblent toutefois plus naturelles (ou acceptables). Selon Rothstein, le pronom it a donc bien un référent non spécifié et la non spécification n'implique pas que le pronom n'a pas de référence ou d'apport sémantique. Selon elle, le pronom est restreint à la forme neutre car il a un référent abstrait, mais ce référent est habituellement spécifique (comme l'indiquent les gloses avec un syntagme nominal défini). De plus, le fait que ces pronoms peuvent parfois être réalisés comme sujet au passif (comme dans (41-43) ci-dessous, empruntés à Rothstein 1995) serait un indice de leur référentialité :

41. It was rushed, but we finally got the job done.

42. It was kept up for a long time.

43. It's being fought out with the management right now. ${ }^{12}$

Rothstein (1995) considère ainsi que, si certains exemples ne sont pas clairement référentiels (it dans Damn it! pouvant être considéré comme un explétif, d'où le parallèle avec Damn!, ou un pronom référentiel, proche de Damn this place!), le doute ne concerne que quelques exemples idiomatiques et non productifs, et ne remet pas en question que dans la majorité des cas it est bien un objet pronominal dénotant une entité spécifique spécifiée contextuellement, dont la portée de dénotation est déterminée par les propriétés sémantiques et thématiques du verbe.

L'hypothèse est alors que it est un quasi-argument, sous-catégorisé, et avec un contenu idiomatique. Une analyse similaire est défendue par Bolinger (1977), dans un autre cadre théorique: si it n'était qu'un explétif, il ne pourrait pas faire l'objet d'un questionnement, or il semble possible d'interroger sur le référent du pronom :

44. Did you have it out with him yet? - Have what out with him?

45. I was disappointed by how easily they bought it. - Bought what?

L'hypothèse d'un it explétif en position d'objet a ainsi fait l'objet de débats entre syntacticiens générativistes. ${ }^{13}$ 
69 Enfin, nous examinerons ici le it de clivée, illustré en (2): What is it that drives you in your life? Ce pronom a également fait l'objet de controverses, puisque deux analyses divergentes ont été avancées : it a été analysé, soit comme un explétif, soit comme un pronom référentiel (Han \& Hedberg 2008). Ces hypothèses dépendent de l'analyse de l'ensemble de la structure.

70 L'hypothèse de it explétif est associée à l'analyse de la subordonnée de clivée comme ayant une relation (de prédication) directe avec l'élément clivé (Jespersen 1937, Chomsky 1977, Williams 1980, Kiss 1998). Un argument en faveur de cette hypothèse est que les accords marquent une connexion entre le constituant clivé et la subordonnée :

46. It is John and Mary that like Pete.

46 '. *It is John and Mary that likes Pete.

$46 "$. *It are John and Mary that like Pete. ${ }^{14}$

71 Ces données favorisent l'analyse du constituant clivé comme adjoint à ou extrait de la subordonnée. Dans l'analyse en tant qu'explétif, défendue par Kiss (1998), ou Lambrecht (2001), le pronom it est supposé être un explétif généré en position sujet, alors que la subordonnée de clivée est sémantiquement liée au constituant clivé par une relation prédicative. Cette hypothèse est motivée par le lien sémantique étroit entre l'expression focalisée et le vide syntaxique dans la subordonnée (variable dont la valeur est identifiée par l'élément focal). La subordonnée de clivée est alors sœur du verbe copule ou d'une tête fonctionnelle encodant le focus. Cette hypothèse est motivée syntaxiquement : comme le notent Delahunty (1984), Hedberg (2000) et Han \& Hedberg (2008), entre autres, certaines données indiquent que le focus forme un constituant syntaxique avec la subordonnée clivée qui suit. En effet, comme l'illustrent les exemples suivants de Han \& Hedberg (2008), l'ellipse peut concerner l'ensemble que forment l'élément focal et la subordonnée ; de même, le focus et la subordonnée peuvent subir un mouvement.

47. I said it should have been [Bill who negotiated the new contract], and it should have been _..

48. It must have been [Fred that kissed Mary] but [Bill that left with her].

49. It could have been - and it should have been - Bill who negotiated the new contract.

50. It must have been, in my opinion, the cyanide that did it.

51. It must have been Fred that kissed Mary but Bill that left with her.

72 Par conséquent, l'hypothèse d'un it explétif est associée à une analyse de la subordonnée de clivée comme une forme de subordonnée relative habituelle.

73 Toutefois cette hypothèse n'est pas entièrement satisfaisante: si it était un simple explétif, il devrait être impossible de remplacer it par this ou that. Or ce remplacement est (marginalement) possible, comme l'indiquent des exemples tels que This is not Iowa we're talking about (Han \& Hedberg 2008). C'est pourquoi l'hypothèse d'un it de clivée pronom a été défendue.

Avec l'hypothèse de it pronom, la subordonnée de clivée a une relation syntaxique et sémantique directe avec $i t$, et est réalisée après le constituant clivé par extraposition, ou en formant un constituant discontinu avec le pronom (Emonds 1976, Gundel 1977, Hedberg 2000). Comme l'indique Jespersen (1927), la clivée "it is the wife that decides" signifie en réalité "the wife is the deciding person" : la subordonnée pourrait être analysée comme liée à it plutôt qu'à l'élément focal qui suit la copule. 

et la subordonnée (Gundel 1977, Geluykens 1988, Hedberg 1988, Akmajian 1970). Elle est justifiée par les différences entre subordonnées de clivées et relatives (restrictives) habituelles : comme le note Jespersen (1927), l'élément focal peut être tellement défini qu'il est impossible de le restreindre davantage (dans des exemples tels que it was the battle of Waterloo that decided the fate of Europe.) Declerck (1988: 152) remarque également que la subordonnée de clivée peut suivre un "antécédent " à référence unique tel qu'un pronom personnel ou un nom propre (It is me that they invited), ce que ne peut jamais faire une relative (*They were thinking of me that they could invite).

Les deux approches des clivées présentent donc des limites. Par conséquent l'analyse du it de clivée en tant que pronom comme celle en tant qu'explétif semblent toutes deux partiellement motivées.

Comme le montre cette dernière section, dans l'analyse des idiomes comme dans celle des clivées, les discussions ont souvent impliqué un questionnement sur le possible contenu sémantique de it. Dans les emplois examinés ici, it se démarque clairement du fonctionnement habituel du pronom anaphorique, d'où la fréquente remise en question de l'idée que ce it est référentiel. Toutefois, comme le rappelle Chomsky (1995), it porte le cas et des traits de personne, nombre et genre (alors que there ne porte que des traits de cas) : il se démarque ainsi d'un simple outil remplissant une fonction syntaxique (placeholder), et il paraît envisageable que, même s'il n'est pas anaphorique, il pourrait conserver une forme de référentialité (vague).

\section{Conclusions}

L'examen des pronoms non anaphoriques soulève des questions théoriques cruciales, et fait appel à des enjeux théoriques centraux des approches génératives classiques. En effet, l'étude de ces questions permet de revenir sur la nécessité ou non de postuler des mouvements ou transformations, mais elle permet également de réexaminer le lien entre sélection sémantique et sélection syntaxique, et de s'interroger sur les parallèles possibles (ou nécessaires) entre syntaxe et sémantique. Ainsi, l'examen de ces pronoms permet d'aborder des concepts fondamentaux de la théorie, même s'ils n'ont pas fait l'objet d'un traitement systématique (au même titre que les pronoms anaphoriques, dont l'étude a contribué à la définition des principes essentiels de la théorie du gouvernement et du liage.

\section{BIBLIOGRAPHIE}

Alexiadou, A., Law, P., Meinunger, A. \& Wilder, C. (eds.) (2000). The Syntax of Relative Clauses. Amsterdam / Philadelphia: John Benjamins.

Baker, C.L. 1995. English Syntax. 2nd ed. Cambridge, MA: MIT Press.

Bennis, H. (1986). Gaps and dummies. Dordrecht: Foris.

Corela, HS-35 | 2022 
Bolinger, D. (1973). Ambient it is meaningful too. Journal of Linguistics 9, 261-270.

Bolinger D. (1977). Meaning and Form. London: Longman.

Börjars, K. \& Vincent, N. Objects and OBJ. In Butt, M. \& King, T. H. (eds.) Proceedings of LFG 2008, 150-168. Stanford, CA: CSLI Publications.

Bresnan, J. \& Grimshaw, J. (1978). The syntax of free relatives in English. Linguistic Inquiry 9, 331391.

Burzio, L. (1986). Italian Syntax: A Government-Binding Approach. Dordrecht: Reidel.

Caponigro, I. 2019. In defense of what(ever) free relative clauses they dismiss: A reply to Donati and Cecchetto (2011). Linguistic Inquiry 50, 356-371.

Cheng, L.L.S. (1991). On the Typology of Wh-Questions. PhD dissertation: MIT.

Chomsky, N. (1973). Conditions on Transformations. In Anderson, S. and Kiparsky, P. (eds.) A

Festschrift for Morris Halle, 232-286. New York: Holt, Rinehart \& Winston.

Chomsky, N. (1977). On wh- movement. In Culicover, P.W., Wasow, T. \& Akmajian, A. (eds) Formal Syntax, 71-132. New York: Academic Press.

Chomsky, N. (1981). Lectures on Government and Binding. Studies in Generative Grammar 9. Dordrecht: Foris Publications.

Chomsky, N. (1986). Barriers. Cambridge, MA: MIT press

Chomsky, N. (1995). The Minimalist Program. Cambridge, MA: MIT Press.

Culicover, P.W. \& Jackendoff, R. (2005). Simpler Syntax. Oxford: Oxford University Press.

Deal, A.R. (2009). The origin and content of expletives: Evidence from "selection". Syntax 12(4), 285-323.

Declerck, R. (1988). Studies on Copular Sentences, Clefts and Pseudoclefts. Dordrecht: Foris.

Delahunty, G.P. (1982). Topics in the Syntax and Semantics of English Cleft Sentences. PhD dissertation. Bloomington, IN: Indiana University Linguistics Club.

Diesing, M. (1992). Indefinites. Cambridge, MA: MIT Press.

Donati, C. \& Cechetto, C. (2011). Relabeling heads: A unified account of relativization structures. Linguistic Inquiry 42, 519-560.

Kiss, K.É. (1998). Identificational focus versus information focus. Language 74, 245-273.

Kiss, K.É. (2002) The EPP in a Topic-prominent Language. In Svenonius, P. (ed.), 107-124. New York: Oxford University Press.

Emonds, J.E. (1976). A Transformational Approach to English Syntax. New York: Academic Press. Groos, A. \& Riemsdijk, H.C. van. (1981). Matching effects in free relatives: A parameter of core grammar. In Belletti, A., Brandi, L. \& Rizzi, L. (eds). Theory of Markedness in Generative Grammar. Proceedings of the 1979 GLOW Conference, 171-216. Pisa: Scuola Normale Superiore.

Gundel, J.K. (1977). Where do cleft sentences come from? Language 53, 543-559.

Haegeman, L. (1991). Introduction to Government and Binding Theory. Oxford: Blackwell.

Hale, K. \& Keyser, J. (2000). There-insertion unaccusatives. Ms. MIT.

Han, C. \& Hedberg, N. (2008). Syntax and semantics of it-clefts: A Tree Adjoining Grammar analysis. Journal of Semantics 25, 345-380. 
Hedberg, N. (2000). The referential status of clefts. Language 76, 891-920.

Hoekstra, T. (1983). The distribution of sentential complements. In Bennis, H. \& van Lessen Kloeke, W.U.S. (eds), Linguistics in the Netherlands 1983, 93-103. Dordrecht: Foris.

Huddleston, R. (1984). Introduction to the Grammar of English. Cambridge: Cambridge University Press.

Jespersen, O. (1927). A Modern English Grammar, vol. 3. London: Allen \& Unwin.

Jespersen, O. (1937). Analytic syntax. London: Allen \& Unwin.

Johnson, G. (2015). The morphosyntax of whatever in free relatives: Variation and optionality in Appalachian English. Presentation at the LSA 2015 Annual Meeting, January 8-11, 2015, Portland, OR.

Lambrecht, K. (2001). A framework for the analysis of cleft constructions. Linguistics 39(3), 463516.

Levin, B. (1993). English Verb Classes and Alternations: A Preliminary Investigation. Chicago: University of Chicago Press.

Perlmutter, D.M. (1983). Personal vs. impersonal constructions. Natural Language and Linguistic Theory, 1(1), 141-200.

Pollard, C. \& Sag, I.A. (1994). Head-Driven Phrase Structure Grammar. Chicago: University of Chicago Press.

Postal, P.M. \& Pullum, G.K. (1988). Expletive noun phrases in subcategorized positions. Linguistic Inquiry 19(4), 635-670.

Radford, A. (1988). Transformational Grammar: A First Course. Cambridge: Cambridge University Press.

Rosenbaum, P.S. (1968). The Grammar of English Predicate Complement Constructions. Cambridge, MA: MIT Press.

Ross, J.R. (1967). Constraints on Variables in Syntax. PhD dissertation. Cambridge, MA: MIT.

Rothstein, S.D. (1995). Pleonastics and the interpretation of pronouns. Linguistic Inquiry 26(3), 499-529.

Svenonius, P. (ed.) Subjects, Expletives, and the EPP. New York: Oxford University Press.

Trotta, J. (2004). Comments on vacuous subject movement. In Bergh, G., Herriman, J. \& Mobärg,

M. (eds.) An International Master of Syntax and Semantics: Papers presented to Aimo Seppänen on the occasion of his 75th birthday, 217-238. Gothenburg Studies in English 88. Göteborg: Göteborg University.

Riemsdijk, H.C. van. (2006). Free relatives. In Everaert, M. \& Riemsdijk, H.C. van (eds.) The Blackwell Companion to Syntax, vol.2, 338-382. Oxford: Blackwell.

Williams, E. (1980). Predication. Linguistic Inquiry 11(1), 203-238.

\section{NOTES}

1. Les exemples numérotés donnés à analyser dans le texte initial sont indiqués par des chiffres entre parenthèses, tandis les exemples introduits dans la discussion qui suit (extraits d'ouvrages sur les pronoms anaphoriques) sont introduits par des chiffres suivis d'un point. 
2. La forme $[t]$ correspond à la trace laissée par l'élément wh- dans sa position initiale putative. Les traces ne sont indiquées que dans les parties de la discussion précisant les contraintes sur les mouvements.

3. Ces énoncés sont d'une acceptabilité discutable en anglais standard, comme le notent Radford (1988: 500) et Trotta (2004: 4).

4. L'agrammaticalité de 4 . est due au fait que le verbe interview ne sélectionne que des objets nominaux (DPs et non PPs).

5. On notera que ${ }^{*}$ Der Hans hat zurückgegeben, das Geld, das er gestohlen hat est exclu car le déplacement en position finale de NPs lourds est impossible en allemand (pas de heavy NP shift).

6. [ _ ] représente la tête (putative) de la relative.

7. Il n'est pas certain que it soit explétif dans cet exemple. Ce pronom pourrait avoir une référence vague (et pourrait être remplacé par the situation / the picture). Toutefois, un rapprochement avec d'autres emplois de it 'explétif' en position de complément (e.g. live it up) nous paraît pertinente dans la mesure où, dans un ensemble de cas, une hésitation est possible entre lecture référentielle vague et analyse en tant qu'explétif, des analyses concurrentes étant défendues selon les approches théoriques adoptées.

8. Pour rappel, les verbes inergatifs sont des verbes dont le sujet est supposément généré en position sujet, alors que l'argument (non agentif) des verbes inaccusatifs est généré en position d'objet (mais n'est pas au cas accusatif car réalisé en structure de surface en tant que sujet). Les verbes inaccusatifs sont souvent présentés comme similaires à des verbes au passif.

9. Les verbes inchoatifs (tels que break, freeze ou boil) peuvent désigner le début d'un événement, ces verbes peuvent souvent entrer dans une alternance causative : utilisés transitivement, ils ont pour sujet la cause de l'événement (Bob opened the door), alors que lorsqu'ils sont utilisés intransitivement, seul le thème est réalisé (The door opened).

10. Plus précisément : selon Deal (2009), le fait qu'il est possible d'observer un mouvement de there, d'une position enchâssée à la position de sujet de la principale, et non une fusion (merge) d'un there en position enchâssée avec un there dans la principale, reflèterait une tendance générale à favoriser le mouvement plutôt que la fusion d'éléments indépendants. Cela reflèterait une tendance générale (move over merge) qui explique également le nombre limité de pronoms résomptifs dans diverses langues.

11. EPP: Extended Projection Principle, principe de projection étendue selon lequel toute proposition doit voir sa position sujet instanciée, même lorsque le verbe n'assigne pas de rôle thématique - d'où la présence de l'explétif it sujet de verbes météorologiques : it's raining.

12. Il est toutefois possible que certains de ces énoncés n'impliquent pas un pronom anaphorique habituel. Par ailleurs, la passivation n'est pas toujours possible : I'll have to wing it / It will have to be winged.

13. Un débat similaire sur le it d'extraposition du sujet a opposé des auteurs supposant qu'il s'agit d'un simple explétif à des auteurs supposant que it est référentiel (Hoekstra 1983, Bennis 1986), ou qu'il est co-indexé avec un CP (Rosenbaum 1968). Nous ne développons pas ce point, car le texte ne contient pas d'exemples d'extraposition du sujet.

14. Toutefois, dans les clivées prédicationnelles, on peut observer un accord du pronom et de la copule avec le constituant clivé : ex. They're just fanatics who are holding him / Those are kids who beat John (Han \& Hedberg 2008). 


\section{RÉSUMÉS}

La distribution des pronoms anaphoriques est un enjeu central dans un ensemble de travaux en grammaire générative transformationnelle classique, les contraintes sur les pronoms anaphoriques étant un des thèmes centraux de la théorie du gouvernement et du liage. Les pronoms non anaphoriques n'ont pas fait l'objet d'un traitement aussi systématique, mais leur analyse repose sur des concepts centraux à l'analyse transformationnelle. D'une part cette analyse implique une réflexion sur le mouvement (des pronoms wh-) et motive la distinction entre structure de surface et structure profonde. L'étude des différents pronoms wh- appelle par ailleurs un questionnement sur le fonctionnement (nominal ou propositionnel) des constituants en wh-. D'autre part l'analyse des pronoms explétifs (deuxième type de pronoms non anaphoriques) exige une réflexion sur l'autonomie de la syntaxe, ou les décalages éventuels entre syntaxe et sémantique.

The distribution of anaphoric pronouns has been a crucial issue in 'mainstream' transformational grammars (in particular in the government and binding framework). Non-anaphoric pronouns have not been as extensively examined, but their analysis requires the re-examination of central tenets of transformational approaches. On the one hand the analysis of non-anaphoric pronouns requires a reflection on movement (of wh-pronouns) and motivates the distinction between deep and surface structures. The study of different wh-pronouns also requires a reflection on the more or less nominal characteristics of wh-constituents. On the other hand, the analysis of expletive pronouns (i.e. the second type of non-anaphoric pronouns) triggers a reflection on the autonomy of syntax, or on possible discrepancies between syntax and semantics.

\section{INDEX}

Keywords : transformation, movement, interrogative, relative, expletive

Mots-clés : transformation, mouvement, interrogatif, relatif, explétif

\section{AUTEUR}

\section{ANNE JUGNET}

Université Paris Diderot - CLILLAC-ARP 